\title{
Improved Detection of Helicobacter pylori Infection and Premalignant Gastric Mucosa Using "Site Specific Biopsy": a Randomized Control Clinical Trial
}

\author{
Taweesak Tongtawee ${ }^{1,4 *}$, Chavaboon Dechsukhum ${ }^{3,4}$, Wilairat Leeanansaksiri $^{5}$ \\ Soraya Kaewpitoon ${ }^{2,4}$, Natthawut Kaewpitoon ${ }^{6,7,8}$, Ryan A Loyd ${ }^{2,4}$, Likit \\ Matrakool ${ }^{1,4}$, Sukij Panpimanmas ${ }^{1,4}$
}

\begin{abstract}
Background: Helicobacter pylori infection and premalignant gastric mucosa can be reliably identified using conventional narrow band imaging (C-NBI) gastroscopy. The aim of our study was to compare standard biopsy with site specific biopsy for diagnosis of $\boldsymbol{H}$. pylori infection and premalignant gastric mucosa in daily clinical practice. Materials and Methods: Of a total of $\mathbf{5 0 0}$ patients who underwent gastroscopy for investigation of dyspeptic symptoms, 250 patients underwent site specific biopsy using C-NBI (Group 1) and 250 standard biopsy (Group 2). Sensitivity, specificity, and positive and negative predictive values were assessed. The efficacy of detecting $\boldsymbol{H}$. pylori associated gastritis and premalignant gastric mucosa according to the updated Sydney classification was also compared. Results: In group 1 the sensitivity, specificity, positive and negative predictive values for predicting $\boldsymbol{H}$.pylori positivity were $95.4 \%, 97.3 \%, 98.8 \%$ and $90.0 \%$ respectively, compared to $92.9 \%$, 88.6\%, 83.2\% and 76.1\% in group 2. Site specific biopsy was more effective than standard biopsy in terms of both $H$. pylori infection status and premalignant gastric mucosa detection $(\mathrm{P}<0.01)$. Conclusions: Site specific biopsy using $\mathrm{C}-\mathrm{NBI}$ can improve detection of $\boldsymbol{H}$.pylori infection and premalignant gastric mucosa in daily clinical practice.
\end{abstract}

Keywords: Site specific biopsy - standard biopsy - Helicobacter pylori infection - premalignant gastric mucosa

Asian Pac J Cancer Prev, 16 (18), 8487-8490

\section{Introduction}

Gastroscopy with biopsy of the gastric mucosa for rapid urease testing and histopathological examination remain the standard for diagnosis of Helicobacter pylori associated gastritis in daily clinical practice including in Thailand. Since the discovery of $H$.pylori in 1983, strong evidence has indicated that $H$. pylori infection plays an important role in the pathogenesis of chronic gastritis, gastric ulcer disease, gastric atrophy, intestinal metaplasia and gastric malignancy change (Komoto et al., 1998; Mihara et al., 1999).

Although gastroscopic features of $H$.pylori associated gastritis have been reported in the literature, endoscopic diagnosis of $H$. pylori infection of the gastric mucosa by using conventional endoscopy and an Indigo carmine contrast method may be possible (Takahiro Kato et al., 2013). However are there the controversies about whether $H$. pylori associated gastritis that can be diagnosed by gastroscopic features alone. The Narrow Band Imaging system (NBI) is an endoscopic imaging technique for enhanced visualization of mucosal microscopic structuress and capillaries in the superficial mucosal layer. Images are obtained by using Narrow Band red, blue, and green filters, which are different from conventional red-green-blue filters (Gono et al., 2004). With the development of high resolution endoscopes; it became possible to investigate the microstructure of the gastrointestinal tract. Recently, the appearance of normal $H$. pylori negative stomach as well as alterations induced by $H$.pylori associated gastritis have been described (Yagi et al., 2002).

High resolution NBI endoscopy is useful for predicting H.pylori infection and the histological severity of gastritis and is valuable for predicting gastric atrophy in the entire stomach (Tahara et al., 2009). Furthermore, high resolution endoscopy with narrow band imaging has good efficacy for detecting early gastric cancer (Hang et al., 2015; Zhang et al., 2015). The Kyoto global consensus report on H.pylori gastritis suggested that atrophic mucosa and intestinal metaplasia can be accurately detected by imageenhanced endoscopy, after appropriate training (Kentaro Sugano et al., 2015). However, in daily clinical practice,

${ }^{1}$ Department of Surgery, ${ }^{3}$ Pathological Unit, Institute of Medicine, ${ }^{2}$ Family Medicine and Community Medicine, ${ }^{6}$ Parasite Disease Research Unit, ${ }^{5}$ Institute of Science, Suranaree University of Technology, ${ }^{4}$ Suranaree University of Technology Hospital, ${ }^{7}$ Division of Research Clinic, Suranaree University of Technology Hospital, ${ }^{8}$ Faculty of Public Health, Vongchawalitkul University, Nakhon Rachasima, Thailand*For correspondence: taweesak.t@sut.ac.th 


\begin{tabular}{llccc}
\hline Histologic Properties & \multicolumn{1}{c}{ Definition } & \multicolumn{2}{c}{ Grading } \\
\cline { 3 - 5 } & & mild & moderate & severe \\
\hline Chronic inflammation & Lymphocyte and plasma cell in lamina propria & $1+$ & $2+$ & $3+$ \\
Neutrophil activation & Neutrophilic infiltration in lamina propria or superficial epithelium & $<1 / 3$ & $1 / 3-2 / 3$ & $>2 / 3$ \\
Glandular atrophy & Loss of corpus and antral glands & $1+$ & $2+$ & $3+$ \\
Intestinal metaplasia & Intestinal metaplasia of mucosal epithelium & $<1 / 3$ & $1 / 3-2 / 3$ & $>2 / 3$ \\
Helicobacter pylori & Helicobacter pylori intensity & $1+$ & $2+$ & $3+$ \\
\hline
\end{tabular}

high resolution endoscopy seems to not be feasible, because it takes more examination time and needs more training experience of the endoscopist. According to our study for Conventional Narrow Band Imaging gastroscopy (C-NBI), the results show good efficacy for diagnosis of $H$. pylori infection and good correlation to the histopathology of the gastric mucosa (Taweesak et al., 2015). The main purpose of this study was to compare the efficacy of site specific biopsy with standard biopsy for the diagnosis of $H$. pylori associated gastritis and premalignant gastric mucosa in daily clinical practice.

\section{Materials and Methods}

\section{Patients}

A total of 500 patients who underwent elective gastroscopy for the investigation of dyspeptic symptoms were invited to participate in the study from November 2014 to November 2015 at the Endoscopic unit, Department of Surgery, Suranaree University of Technology Hospital (SUTH), Institute of Medicine, Suranaree University of Technology, Nakhonrachasima, Thailand. We randomized the patients into 2 groups using the Random Number Generator by SPSS for Windows (version 16.0; SPSS, Chicago, IL, USA).The following exclusion criteria were applied: age below 18 or above 70 years, Helicobacter pylori eradication treatment in the previous 2 months, significant medical illnesses history of previous gastric surgery, of the use of antimicrobials or gastrointestinal medications like PPIs, H2blockers or bismuth compounds within the previous 2 months. All patients provided informed consent, and the study was approved by the institutional review board of Suranaree University of Technology, Nakhonrachasima, Thailand. EC-57-36

Diagnosis of helicobacter pylori associated gastritis

A diagnosis of H.pylori infection was made if $H$.pylori morphology were seen on histopathological examination and the rapid urease test during gastroscopy was positive.

\section{Biopsy specimens}

The gastroscopic procedures were performed using an upper GI video endoscope (Olympus EVIS EXERA III, CV-190).The whole stomach was examined first with conventional endoscopy. After the whole stomach mucosa was observed we chose sites according to previous studies (Taweesak et al., 2015).

\section{Standard biopsy}

Biopsies were taken from five standardized intragastric

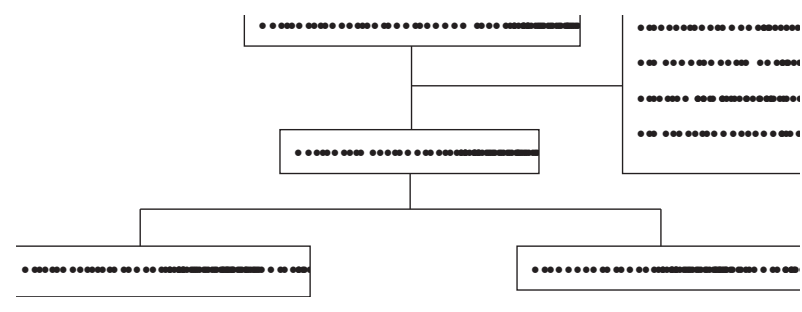

Figure 1. Flow Diagram Showing Numbers of Patients Enrolled

Table 2. Patient Baseline Demographics Data

\begin{tabular}{lc}
\hline Demographics data & $\mathrm{n}=500$ \\
\hline Male/female (n) & $148 / 352$ \\
Mean age (years) & 45.2 \\
Mean follow-up time,( day) & $168 \pm 4$ \\
Peptic ulcer disease (GU/DU) & $16 \%$ \\
Non ulcer gastritis/duodinitis & $73 \%$ \\
Gastroesophageal reflux disease & $11 \%$ \\
\hline
\end{tabular}

Table 3. Helicobacter pylori Infection Status among Biopsy Techniques

\begin{tabular}{lcc}
\hline \multirow{2}{*}{ Biopsy technique } & \multicolumn{2}{c}{ Helicobacter pylori infection status } \\
\cline { 2 - 3 } & $\begin{array}{c}\text { Non-infected } \\
\text { subjects(HP -) }\end{array}$ & $\begin{array}{c}\text { infected } \\
\text { subjects(HP +) }\end{array}$ \\
\hline Site specific biopsy & $31.6(79 / 250)$ & $68.4(171 / 250)$ \\
Standard biopsy & $32.8(82 / 250)$ & $67.2(168 / 250)$ \\
\hline
\end{tabular}

$\mathrm{P}<0.01$

Table 4. Premalignant Gastric Mucosa (Gastric Atrophy, Intestinal Metaplasia) among Biopsy Techniques

\begin{tabular}{lcc}
\hline Biopsy technique & \multicolumn{2}{c}{ Premalignant gastric mucosa } \\
\cline { 2 - 3 } & Gastric atrophy & Intestinal metaplasia \\
\hline Site specific biopsy & $6.8(17 / 250)$ & $4.8(12 / 250)$ \\
Standard biopsy & $5.6(14 / 250)$ & $3.6(9 / 250)$ \\
\hline
\end{tabular}

$\mathrm{P}<0.01$

locations. 1: antrum (four quadrants), 2: incisura angularis, 3: corpus greater curvature, 4: corpus lesser curvature, 5: cardia (CM den Hoed et al., 2013)

Site specific biopsy

Four biopsy samples were taken directly from the observation sites by using C-NBI endoscopy. Two samples were sent for histological analysis and 2 were used for rapid urease testing on site (ProntodyleR, GASTREX, France)

Histological analysis

Specimens for histological analysis were placed in 
Improved Detection of $\boldsymbol{H}$. pylori Infection and Premalignant Gastric Mucosa Using "Site Specific Biopsy" Table 5. Diagnostic Value of Endoscopic Features for Helicobacter pylori Gastritis

\begin{tabular}{lcccc}
\hline Endoscopic technique & Sensitivity $(\%)$ & Specificity $(\%)$ & PPV $(\%)$ & NPV $(\%)$ \\
\hline Site specific biopsy & 95.42 & 97.29 & 98.82 & 90 \\
Standard biopsy & 92.98 & 88.6 & 83.24 & 76.08 \\
\hline
\end{tabular}

*PPV, positive predictive value; NPV, negative predictive value

Table 6. Inter- and Intra-observer Agreement

\begin{tabular}{lccccc}
\hline & \multicolumn{2}{c}{ Interobserver agreement } & & \multicolumn{2}{c}{ Intraobserver agreement } \\
\cline { 2 - 3 } Endoscopic technique & \% agreement & k value $(95 \% \mathrm{CI})$ & & \% agreement & $\mathrm{k}$ value (95\% CI) \\
\hline Site specific biopsy & 97.6 & $0.97(0.96-0.98)$ & & 85.6 & $0.85(0.82-0.88)$ \\
Standard biopsy & 95.8 & $0.95(0.93-0.96)$ & & 98.9 & $0.98(0.97-0.99)$ \\
\hline
\end{tabular}

$10 \%$ formalin solution and routinely processed. The hematoxylin and eosin stain and Giemsa stain were used for identification of $H$. pylori. All of the cases were evaluated by 5 pathologist of Bangkok Pathological Laboratory outside Suranaree University according to the Sydney classification (Table1), including evaluation for chronic inflammation, gastric atrophy, intestinal metaplasia, and activity of gastritis.

\section{Image evaluation}

All gastroscopic examinations were digitally recorded and still images of the observation sites were captured for use in the reproducibility study. The selected images were transferred to a software program without distorting brightness, contrast or color balance. A total of 500 pictures from 500 patients were selected for the inter and intra observer agreement study. All endoscopists were blinded to the results of the H.pylori status and histology before reviewing the gastroscopic picture.

\section{Statistical analysis}

The sensitivity, specificity, positive predictive value, negative predictive values were calculated. Student's t -test was used for unpaired parametric data and chi-square test was used for the comparison of nonparametric data. A P-value less than 0.05 were considered significant. All statistical analyses were performed using the SPSS, version 16.0 (SPSS Inc., Chicago, IL, USA).The $x$ value was calculated for inter and intra observer variability. $x$ values below 0.4 indicated poor agreement, values between 0.4 and 0.6 represent moderate agreement, values between 0.6 and 0.8 represented substantial agreement, and values greater than 0.8 corresponded to excellent agreement.

\section{Symptoms and safety evaluation}

The study was performed in accordance with good clinical practice and the guidelines of the Declaration of Helsinki. All patients provided written informed consent and the study protocol was approved by the Ethics Committee for Research Involving Human Subjects Suranaree University of Technology (EC-57-36).

\section{Results}

A total of 500 consecutive patients (148 men, 352 women; mean age 45.2 years, range 19-69 years) were enrolled in the study from November 2014 to November 2015 (Figure 1). Mean follow-up time was $168 \pm 4$ days. Peptic ulcer disease (GU/DU) was $16 \%$, non ulcer gastritis/duodinitis was $73 \%$, and gastroesophageal reflux disease was $11 \%$ (Table 2). The 250 patients in the group 1 had performing site specific biopsy performed and the 250 patients in group2 had standard biopsy performed. In group 1:sensitivity, specificity, positive and negative predictive values for predicting $H$. pylori positive were $95.42 \%, 97.29 \%, 98.82 \%$ and $90.0 \%$ respectively. In group 2:sensitivity, specificity, positive and negative predictive values for predicting $H$. pylori positive were $92.92 \%, 88.60 \%, 83.24 \%$ and $76.08 \%$ respectively. $H$. pylori infection status, premalignant gastric mucosa (Gastric atrophy, Intestinal metaplasia), diagnostic value of endoscopic features for H. pylori gastritis, Inter and intraobserver agreement are summarized in Table 3 to Table 6 respectively.

\section{Discussion}

Many endoscopists believe that a diagnosis of $H$. pylori associated gastritis can be made by the gross appearance of the gastric mucosal morphologic pattern on gastroscopy. The results of our study that examined this hypothesis suggested that gastroscopic mucosal morphologic pattern using Conventional Narrow Band Imaging gastroscopy (C-NBI) such as regular arrangement of collecting venules, cone-shaped gastric pits, rod-shaped gastric pits with prominent sulci, ground glass like morphology and dark brown patches with bluish margin or irregular border can reliably identify $H$. pylori associated gastritis and premalignant gastric mucosa (Taweesak et al., 2015). In daily clinical practice; the problem for the diagnosis of $H$. pylori infection and premalignant gastric mucosa is choosing the specific area for sampling the gastric mucosa because the histopathology is therefore currently considered to be the gold standard for detecting Helicobacter pylori associated gastritis and the presence of premalignant lesions of the gastric mucosa. The reliability of detecting $H$. pylori gastritis and related conditions such as atrophy and intestinal metaplasia by "blind biopsy" sampling of gastric mucosa depends on the site, number, and size of biopsy specimens (CM den Hoed et al., 2013). In daily clinical practice, this technique is this technique is, resulting in sampling errors, missed pathology, and unnecessary work and costs for the 
pathology departments. The progress in high resolution gastroscopy has allowed direct observation of surface microstructure of the gastrointestinal mucosa. High resolution gastroscopy with NBI has been shown to have a high accuracy for diagnosing early gastric cancer and is an effective screening tool to achieve superior accuracy (Hang et al., 2015). A recent Meta-Analysis, demonstrated that the diagnostic efficacy of high resolution endoscopy with Narrow-Band Imaging for gastric neoplasms has a high diagnostic value for gastric neoplasms and a high specificity (Xiuhe et al., 2015; Ying-Ying et al., 2015). However, in daily clinical practice, high resolution gastroscopy seems to not be feasible because it takes more examination time and requires more training and experience of the endoscopist. The diagnostic advantages and validation of using the gastric mucosal morphologic pattern as demonstrated in this study will hopefully be an impetus for the acceptance of this novel technique for selection of specific areas for biopsy of gastric mucosa. The results of our study indicate that site specific biopsy gastric using C-NBI gastroscopy significantly appears to be a significantly better tool than using the standard blind biopsy technique from five standardized intragastric locations. Furthermore, C-NBI gastroscopy is able to identify high yield areas for gastric atrophy and intestinal metaplasia, and enables "site specific biopsy", potentially eliminating the need for random biopsies. C-NBI gastroscopy may reduce sampling error and decrease the need for biopsies, that add to the cost of the procedure.

In conclusion, considering the unsatisfactory performance of standard biopsy and the limitations of high resolution endoscopic imaging technique, our study suggests that site specific biopsy by using C-NIB gastroscopy improves the detection of $H$.pylori infection and premalignant gastric mucosa in daily clinical practice.

\section{Acknowledgements}

This study was supported by a grant for medical investigation from the Nation Research Council (NRC) and was approved by the Ethics Committee for Research Involving Human Subjects Suranaree University of Technology (EC-57-36) and TCTR20141211002.

\section{References}

CM den Hoed, IL Holster, LG Capelle, et al (2013). Follow-up of premalignant lesions in patients at risk for progression to gastric cancer. Endoscopy, 45, 249-56.

Gono K, Obi T, Yamaguchi M, et al (2004). Appearance of enhanced tissue features in narrow-band endoscopic imaging. J Biomed Opt, 9, 568-77.

Hang Yu, Ai-Ming Yang, Xing-Hua Lu, et al (2015). Magnifying narrow-band imaging endoscopy is superior in diagnosis of early gastric cancer. World J Gastroenterol, 21, 9156- 62.

Kato T, Yagi N, Kamada T, et al (2013). Diagnosis of Helicobacter pylori infection in gastric mucosa by endoscopic features: a multicenter prospective study. Dig Endosc, 25, 508-18.

Kentaro Sugano, Jan Tack, Ernst J Kuipers, et al (2015). Kyoto global consensus report on Helicobacter pylori gastritis. Gut, 64, 1353- 67.

Komoto K, Haruma K, Kamada T, et al (1998). Helicobacter pylori infection and gastric neoplasia: correlations with histological gastritis and tumor histology. Am J Gastroenterol, 93, 1271-6.

Lv X, Wang C, Xie Y, Yan Z, et al (2015). Diagnostic efficacy of magnifying endoscopy with narrow-band imaging for gastric neoplasms: a meta-analysis. PLoS ONE, 10, 1-12.

Mihara M, Haruma K, Kamada T, et al (1999). The role of endoscopic findings for the diagnosis of Helicobacter pylori infection: evaluation in a country with high prevalence of atrophic gastritis. Helicobacter, 4, 40-8.

Sugano K, Tack J, Kuipers EJ, et al (2015). Kyoto global consensus report on Helicobacter pylori gastritis. Gut, 64, 1353-67

Taweesak T, Soraya K, Natthawut K, et al (2015). Correlation between Gastric Mucosal Morphologic Patterns and Histopathological Severity of Helicobacter pylori Associated Gastritis Using Conventional Narrow Band Imaging Gastroscopy. Biomed Res Int , 7, 42-8.

Tahara T, Shibata T, Nakamura M, et al (2009). Gastric mucosal pattern by using magnifying narrow-band imaging endoscopy clearly distinguishes histological and serological severity of chronic gastritis. Gastrointestinal Endosc, 70, 246-53.

Yagi K, Nakamura A, Sekine A, et al (2002). Comparison between magnifying endoscopy and histological, culture and urease test findings from the gastric mucosa of the corpus. Endoscopy, 34, 376-81.

Ying-Ying Hu, Qing-Wu Lian, Zheng-Hua Lin, et al (2015). Diagnostic performance of magnifying narrow-band imaging for early gastric cancer: a meta-analysis. World $J$ Gastroenterol, 21, 7884-94.

Zhang Q, Wang F, Chen ZY, et al (2015). Comparison of the diagnostic efficacy of white light endoscopy and magnifying endoscopy with narrow band imaging for early gastric cancer: a meta-analysis. Gastric Cancer, 1, 1-10. 